\title{
Chatting with the neighbors: crosstalk between Rho-kinase (ROCK) and other signaling pathways for treatment of neurological disorders
}

\author{
Niko Hensel ${ }^{1,2}$, Sebastian Rademacher ${ }^{1,3}$ and Peter Claus ${ }^{1,2,3 *}$ \\ ${ }^{1}$ Hannover Medical School, Institute of Neuroanatomy, Hannover, Germany, ${ }^{2}$ Niedersachsen Research Network on \\ Neuroinfectiology, Hannover, Germany, ${ }^{3}$ Center for Systems Neuroscience, Hannover, Germany
}

OPEN ACCESS

Edited by:

Paul Lingor,

University Medicine Göttingen,

Germany

Reviewed by:

Camilla Bellone,

University of Lausanne, Switzerland

Paul H. Ratz,

Virginia Commonwealth University,

USA

${ }^{*}$ Correspondence: Peter Claus,

Hannover Medical School, Institute of

Neuroanatomy, OE 4140,

Carl-Neuberg-Str. 1, 30625 Hannover,

Germany

claus.peter@mh-hannover.de

Specialty section:

This article was submitted to

Neuropharmacology,

a section of the journal

Frontiers in Neuroscience

Received: 28 March 2015

Accepted: 18 May 2015

Published: 02 June 2015

Citation:

Hensel N, Rademacher S and Claus $P$ (2015) Chatting with the neighbors: crosstalk between Rho-kinase (ROCK)

and other signaling pathways for

treatment of neurological disorders.

Front. Neurosci. 9:198.

doi: 10.3389/fnins.2015.00198
ROCK inhibition has been largely applied as a strategy to treat neurodegenerative diseases (NDDs) and promising results have been obtained in the recent years. However, the underlying molecular and cellular mechanisms are not fully understood and different models have been proposed for neurodegenerative disorders. Here, we aim to review the current knowledge obtained for NDDs identifying common mechanisms as well as disease-specific models. In addition to the role of ROCK in different cell types such as neurons and microglia, we focus on the molecular signaling-pathways which mediate the beneficial effects of ROCK. Besides canonical ROCK signaling, modulation of neighboring pathways by non-canonical ROCK-crosstalk is a recurrent pattern in many NDD-model systems and has been suggested to mediate beneficial effects of ROCK-inhibition.

Keywords: spinal muscular atrophy (SMA), amyotrophic lateral sclerosis (ALS), huntington's disease (HD), parkinson's disease (PD), alzheimer disease (AD), multiple sclerosis (MS), neuroinflammation, microglia

\section{Introduction}

An important signaling hub for the regulation of the actin cytoskeleton and myosin-based contractility is the Rho-kinase (ROCK). Signaling events downstream of ROCK are responsible for key neuronal processes in axonogenesis, growth cone dynamics, and stability of synapses. ROCK has a number of downstream targets including profilins and cofilin (via LIM kinase) as actin-binding proteins as well as myosin light chain phosphatase (MLCP) (Figure 1). Upstream of ROCK, Rho-GTP or phosphatidylinositol-4,5-bisphosphate ( $\left.\mathrm{PIP}_{2}\right)$, and phosphatidylinositol(3,4,5)-trisphosphate ( $\mathrm{PIP}_{3}$ ) activate ROCK (reviewed in Tonges et al., 2011). ROCK-activity mainly acts detrimental on clinically relevant outcomes in neurodegenerative diseases (NDDs). This has been elucidated in the recent years by a series of in vivo animal studies: Expression of dominant negative ROCK-isoforms or application of small molecule inhibitors in rodent-models of several NDDs or diseases with a neurodegenerative component impressively ameliorated diseasephenotypes in many studies. Thereby, small molecule inhibitors are of special interest because of their potential translational use in the clinics compared to genetic approaches. Among those, Y27632 has been extensively used in basic science. However, since now there is only a single 


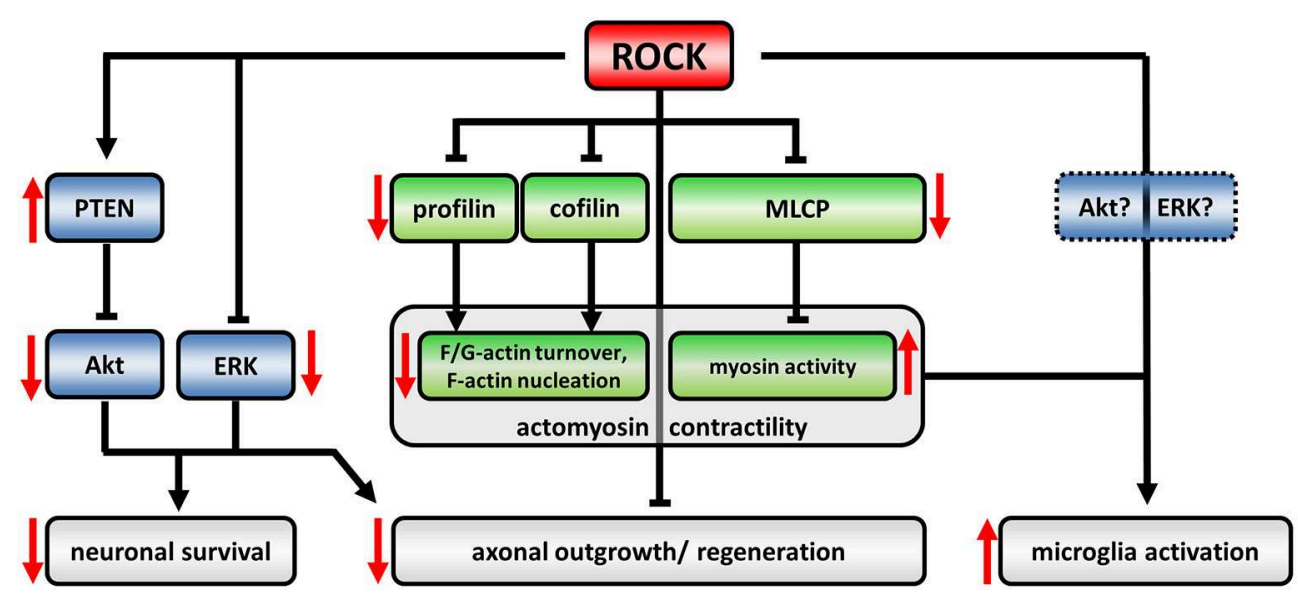

FIGURE 1 | The ROCK-pathway in neurons and microglia. The regulatory network downstream of ROCK is defined by activating (arrows) or inactivating (blunted arrows) interactions. Induction of ROCK-activity leads to an up- or down-regulation of downstream targets, respectively (effects of upregulated ROCK-activity represented by red arrows). ROCK controls actomyosin contractility (canonical signaling, green) as well as Akt- and ERK-activity (non-canonical crosstalk, blue). ROCK-dependent profilin phosphorylation reduces its recycling capacity for globular (G)-actin leading to decreased turnover between filamentous (F) and G-actin. Cofilin-phosphorylation inactivates its F-actin severing activity resulting in less $\mathrm{F}$-actin nucleation. Phosphorylation and inactivation of myosin light chain phosphatase (MLCP) induces myosin activity facilitating retrograde flow of F-actin. Together, those changes lead to a collapsing growth cone and inhibit axonal regeneration processes (reviewed in Lowery and Van Vactor, 2009; Gomez and Letourneau, 2014). ROCK phosphorylates and activates PTEN which in turn inactivates Akt. Similarly, ERK-activity is negatively regulated by ROCK. Neurotrophic Akt- and ERK-pathways are well described agonists of neuronal survival. However, also neuronal morphology is targeted by Akt/ERK signaling thereby influencing neuronal regeneration processes. The role of ROCK activity in microglia-activation is less understood. However, cytoskeletal alterations in ROCK-inhibitor treated cells implicate an involvement of canonical signaling, while ROCK-crosstalk has not been evaluated in microglia yet.
ROCK-inhibitor, Fasudil, which obtained drug approval by Japanese authorities for ischemic stroke treatment (Chen et al., 2013a). Together with the promising results in NDD animal models this hints for broad neuroprotective effects of ROCKinhibition. However, distinct mechanisms are attributable for that.

Here, we aim to review the current literature of ROCKinhibition bringing together different fields of NDDs, thereby identifying common mechanisms including crosstalk with other downstream pathways as well as disease specific effects. Anticipating this, enhanced ROCK-activities have been reported in many in vitro and in vivo NDD models. Thus, ROCK inhibition might at least in part exert its beneficial effects on NDDs by rescuing pathomechanistic changes. Thereby, two dimensions of elevated ROCK-activity have to be taken into account: (i) The pathway dimension, and (ii) the celltype dimension. (i) Canonical ROCK signaling has been extensively studied and associated with the control of actomyosin contractility via phosphorylation of downstream actin-binding proteins such as cofilin or profilin as well as myosin-binding proteins such as myosin light chain phosphatase (reviewed in Gomez and Letourneau, 2014) (Figure 1). In neuronal cells, globally enhanced ROCK-activity leads to growth cone collapse indicating a detrimental role in regenerative, outgrowth related processes (Lowery and Van Vactor, 2009). Despite that, ROCK negatively controls neurotrophic pathway-signaling, which we will term "non-canonical ROCK-crosstalk". Those pathways involve PTEN, Akt, and ERK acting upstream of neuronal survival (Lingor et al., 2008; Takata et al., 2013; Hensel et al.,
2014). Thus, enhanced neuronal ROCK activity in NDDs might inhibit regeneration as well as survival (Figure 1). However, the mechanisms of the crosstalk still lack a detailed molecular description.

(ii) In microglia, ROCK-activity is critically involved in neurodegeneration. Despite microglia, other inflammatory cells are likely involved in neurodegeneration. However, in the context of ROCK-inhibition in NDDs microglia have been most extensively studied. Microglia, also termed "macrophages of the nervous system", have ambiguous roles in NDDs dependent on their activation state (reviewed in Tang and Le, 2015): Activated M1 microglia represent an early reaction to neuronal insults ensuring an inflammatory and neurotoxic environment. However, neurotoxicity might affect healthy neurons inducing a vicious cycle with chronic neuroinflammation (Tang and Le, 2015). In contrast, alternatively activated or deactivated M2-microglia are anti-inflammatory and support tissue and extracellular matrix repair (Tang and Le, 2015). Interestingly, ROCK activity is needed to maintain M1-phenotype as Fasudil-mediated ROCK-inhibition shifts M1-microglia to the M2-state (Zhang et al., 2013). Moreover, activation of the microglial ROCK-axis was associated with cytoskeletal changes and enhanced migration indicating involvement of canonical ROCK-signaling (Bernhart et al., 2010). Additionally, superoxide production was increased (Moon et al., 2013). In turn, microglia express repellents such as chondroitin sulfate proteoglycans (CSPG) which activate ROCK in neurons, thereby inhibiting axonal regeneration (Monnier et al., 2003; Koch et al., 2014). Thus, the benefits of ROCK-inhibition rely on the 
manipulation of cellular and molecular (patho-) mechanisms which are at least in part shared by all NDDs. Those mechanisms can be categorized into (i) support of axonal regeneration, (ii) support of neuronal survival, and (iii) inhibition of detrimental neuroinflammation. Moreover, we and others showed that ROCK-inhibition beneficially interferes with pathological molecular mechanisms specific for a single NDD (Figure 2).

\section{Parkinson's Disease (PD)}

PD is characterized by a loss of dopaminergic neurons within the substantia nigra pars compacta leading to an imbalanced basal ganglia signaling. Clinically, this manifests in severe motor impairments such as tremor, rigor, bradykinesia, and postural instability. Although a number of environmental risk factors have been linked with some cases of sporadic PD,

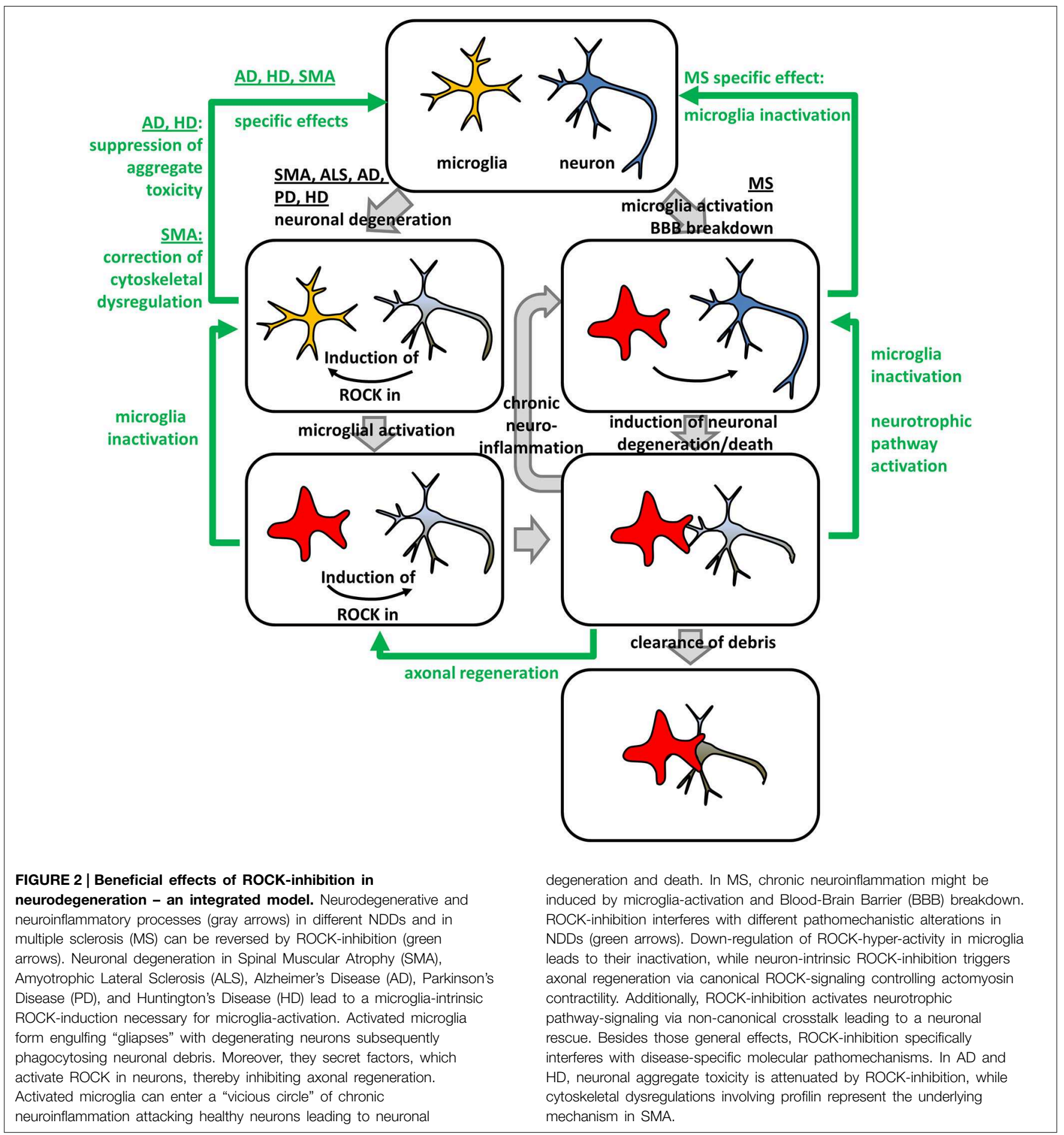


the vast majority still remains of unknown etiology (reviewed in Schiesling et al., 2008). Beside these idiopathic PD cases, several genes have been identified to be involved in familial Parkinsonism. Among those, alpha-synuclein was the first to be identified (Polymeropoulos et al., 1997). Together with autosomal dominant point mutations, gene multiplications of wild-type alpha-synuclein have been linked to an enhanced susceptibility for PD (Singleton et al., 2003; Nishioka et al., 2006). The latter argues for a pathological gain of function mechanism. Interestingly, alpha-synuclein is also a major component of Lewy bodies (LBs) (Spillantini et al., 1997). LBs are neuronal cytoplasmic protein aggregates found in surviving neurons post mortem and represent a central pathological hallmark of idiopathic PD (Gibb and Lees, 1989). This hints for alpha-synuclein as a common pathomechanistic internode in PD-etiology. Supporting this, toxin-induced dopaminergic neurodegeneration, classically employed as PD model, is dependent on alpha-synuclein abundance: Knock-out mice were resistant to 1-methyl-4-phenyl-1,2,3,6-tetrahydropyrine (MPTP)-induced toxicity of dopaminergic cells (Dauer et al., 2002). Importantly, mutant alpha-synuclein transduced neuronal cultures as well as MPTP-based in vivo models displayed a hyperactive ROCK-signaling axis, emphasizing ROCK-upregulation as a common mechanism of genetic and toxin-induced PD-models (Barcia et al., 2012; Villar-Cheda et al., 2012; Tonges et al., 2014b).

Such toxin-induced rodent models have also been extensively employed to study the impact of ROCK-inhibition on clinically relevant outcomes in PD (Barcia et al., 2012; Tonges et al., 2012, 2014b; Tatenhorst et al., 2014; Saal et al., 2015). The severity of dopaminergic neuron damage might thereby be critical for the success of ROCK inhibition: While severely lesioned 6hydroxy dopamine (6-OHDA) animals did not respond to oral Fasudil-application with respect to dopaminergic neuron numbers or motor-phenotype (Tatenhorst et al., 2014), the less severe MPTP-mouse displayed a partial rescue of dopaminergic neuron numbers within the substantia nigra, a partial rescue of TH-fiber density as well as motor-behavior (Tonges et al., 2012). Moreover, an up-regulation of the activity of the pro-survival Akt-pathway was reported in response to co-application with $\mathrm{MPP}+$, the toxic MPTP-metabolite, and Fasudil in vitro (Tonges et al., 2012). As the MPTP-model displays an axonal dyingback pathology preceding neuronal demise (Li et al., 2009a), the authors suggested a dual impact of ROCK-inhibition on (i) axonal regeneration supported by (ii) induction of pro-survival pathways. The first might thereby be mediated by canonical ROCK-signaling controlling actomyosin contractility at the tip of the regenerating axon while the latter might be dependent upon non-canonical ROCK-crosstalk partners such as Akt.

In general, such non-canonical crosstalk is not restricted to Akt, but seems to involve neurotrophic signaling more broadly: In vitro and in vivo results demonstrated increased activities of STAT3, ERK, and Akt - classical neurotrophic pathways - when treated with ROCK-inhibitor Y27632. Importantly, these crosstalk were dependent on neurotrophic pathway activation as they could only be detected under co-treatment with neurotrophic factors such as Ciliary Neurotrophic Factor (CNTF) or Fibroblast Growth Factor 1 and 2 (FGF1, FGF2)
(Lingor et al., 2008; Lin et al., 2009; Hensel et al., 2014). Additionally, we could show that the crosstalk between ROCK and ERK is bi-directional which might have important implications for the pathological situation in NDDs in general: Enhanced neuronal ROCK-activities in NDDs might not only hamper axonal regeneration via changed acto-myosin contractility but also shift neurons toward pro-apoptotic signaling (Hensel et al., 2014).

Interestingly, MPTP-induced reduction of TH+-cell numbers were also partially rescued in primary neuron/glia cultures by application of the ROCK-inhibitor Y27632 while there was no effect on pure neuronal cultures. In contrast, Y27632 was able to rescue neurite lengths independent of microglia abundance (Borrajo et al., 2014). This points toward cell-type specific beneficial roles of ROCK inhibition in PD: Supporting axonal regeneration via neuron-intrinsic pathways as well as inhibition of dopaminergic neuron loss via microglia-dependent mechanisms. The importance of microglia as a target for ROCKinhibition in PD has been further underlined as MPP+-treatment led to an enhanced ROCK-activity in glia-cells in vitro (VillarCheda et al., 2012). Accordingly, MPTP-treatment induced microglial activation leading to an increase in contacts between microglia and dopaminergic neurons in vivo (Barcia et al., 2012). Those contacts, displayed distinct morphological properties with F-actin clusters polarized toward the microglia-neuron contact side engulfing degenerating neurons, similar to the immunological synapse - therefore termed "gliapse" by the authors (Barcia et al., 2012). Importantly, application of Fasudil completely prevented microglial activation and fully rescued TH+-cell numbers (Barcia et al., 2012). However, it is still possible that the in vivo rescue of dopaminergic neurons is at least in part neuron-intrinsically mediated by non-canonical antiapoptotic pathways such as Akt or ERK. Supporting this, an AAV2-mediated knock-down of ROCK2, preferentially expressed in neurons, induced ERK-activity in vitro, partially rescued the number of TH+-cells and mildly improved the motor-phenotype in vivo (Saal et al., 2015). A cell-type-specific ROCK-inhibition in microglia or neurons would therefore be an interesting approach to further clarify the detrimental contribution of microglia versus neuron-intrinsic ROCK-activity in PD.

\section{Multiple Sclerosis (MS)}

MS is a clinically diverse chronic CNS-disease characterized by inflammatory cell plaques in the white matter, loss of myelin and oligodendrocytes as well as gliosis (reviewed inGoldenberg, 2012; Duffy et al., 2014). The etiology of MS is largely unknown. However, together with histopathological findings, genetic risk factors such as variations of the major histocompatibility complex (MHC) (International Multiple Sclerosis Genetics et al., 2011) argue for an important role of neuroinflammation. Blood brain barrier (BBB) breakdown with infiltrating peripheral immune-cells is a characteristic event in the natural history of MS (reviewed in Ortiz et al., 2014). Interestingly, microglia are activated in advance of peripheral immune cell infiltration, underlining the importance of these cells in MS-disease progression (Ponomarev et al., 2005). Alternatively, it was 
suggested that MS is in fact a degenerative disorder (reviewed in Stys et al., 2012) which is supported by axonal damage not exclusively occurring in areas affected by auto-immunity (Trapp et al., 1998). At least, those axonal transections point toward a neurodegenerative component in MS-pathology.

Experimental autoimmune encephalomyelitis (EAE) has been extensively employed as an MS-animal model. Autoimmunization with myelin-sheet protein components such as myelin, oligodendrocyte glycoprotein (MOG), or Proteolipid Protein (PLP) induces pathological features similar to MS including neuroinflammation, demyelination, and neuronal damage (reviewed in Baxter, 2007). Importantly, ROCK upstream effector RhoA was upregulated in MS-patient plaques (Tajouri et al., 2005), and a PLP-induced EAE-mouse model exhibited an enhanced ROCK-activity in the CNS (Sun et al., 2006). Consistently, Fasudil application either in a preventive or therapeutic paradigm reduced clinical severity in different EAEmodels (Sun et al., 2006; Hou et al., 2012). This was accompanied by a reduction of infiltrating inflammatory cells, demyelinated areas, and axonal transactions. Moreover, immune cells isolated from Fasudil-treated mice exhibited a decreased potential for immune response against EAE-inducing antigen, indicating a major role of ROCK-activity in mediation of auto-immunity by inflammatory cells (Sun et al., 2006). However, a chronic disease like MS demands long-term treatments with a minimal amount of side-effects. Thus, alternative application routes combining high CNS-availability with low peripheral abundance are favorable. Interesting results in this context were obtained by intranasal delivery of a Fasudil-derivate in a MOG-induced EAE mouse model (Li et al., 2014b). This treatment-regimen efficiently reduced demyelination, improved clinical scores, and enhanced body weight. Besides a reduction of neuroinflammation, the authors reported elevated BDNF- and NGF-levels suggesting an involvement of neurotrophic pathways by a yet unknown mechanism (Li et al., 2014b). However, this co-occurrence of neurotrophic factor-expression and ROCK-inhibition suggests an induction of pro-survival signals by non-canonical ROCKcrosstalk. Therefore, it is possible that beneficial effects of ROCK-inhibition in EAE are not only based on inhibition of neuroinflammation but are also mediated neuron-intrinsically.

\section{Alzheimers Disease (AD)}

The central clinical manifestations of $\mathrm{AD}$ are progressive cognitive deficits, namely memory, and learning disabilities. The neuropathological picture includes hippocampal atrophy with neuronal loss and reduced synaptic contacts. However, the characteristic histological findings defining $\mathrm{AD}$ are extracellular senile plaques and intraneuronal neurofibrillary tangles (reviewed in Perl, 2010). While the first is composed of amyloid$\beta$ (A $\beta$ ) peptides, the latter consists of hyper-phosphorylated tau-protein (reviewed in Bloom, 2014). The causal nature of $A \beta$ pathology is still under debate and findings of $A \beta$-accumulations without clinical manifestations question a strict relationship between $\mathrm{A} \beta$-plaques formation and $\mathrm{AD}$-symptoms for late-onset sporadic AD (reviewed in Moreno-Trevino et al., 2015). In contrast, mutations in the amyloid precursor protein (APP) as well as in presenilin 1 and 2 (PSEN1, 2) which participate in APP-processing cause hereditary early onset AD (Selkoe, 2001; Haass et al., 2012). This hints for an etiology based on $A \beta$ synthesis and secretion pathways. Thus, idiopathic and familial $\mathrm{AD}$ might differ in etiology, however, idiopathic AD-brains display enhanced neuronal ROCK2-protein levels (Herskowitz et al., 2013) as well as an altered subcellular distribution of its upstream effector RhoA which is a characteristic in common with genetic AD-mouse models (Huesa et al., 2010). Moreover, several studies demonstrated a role of the ROCK-pathway in $\mathrm{A} \beta$-synthesis and secretion (Zhou et al., 2003; Herskowitz et al., 2013). Thus, the ROCK-pathway is a common target for both, idiopathic and familiar $\mathrm{AD}$.

Intracerebroventricular $A \beta$-injections in rats lead to severe cognitive impairments, neuronal damage and an inflammatory response (Song et al., 2013) and can be employed as an ADmodel reflecting $A \beta$-downstream effects. Interestingly, Fasudilapplication rescued spatial learning and memory deficits as well as apoptosis phenotype in the AD-rat hippocampus. Moreover, inflammatory response indicated by enhanced IL- $1 \beta$, TNF $\alpha$, and NFKB-production was reduced back to normal levels (Song et al., 2013). Those effects might be mechanistically attributable to an inhibition of neuroinflammation or to an induction of anti-apoptotic neurotrophic pathways via non-canonical ROCKcrosstalk partners; however, the latter has not been tested in this AD-model yet.

Despite its action on neuroinflammation, several studies revealed a direct molecular role of the ROCK pathway within the $A \beta$-biosynthesis pathway. Plasma membrane bound APP is subjected to the non-amyloidogenic pathway by $\alpha$-secretase cleavage leading to non-toxic $\operatorname{sAPP} \alpha$. When internalized, APP can be subsequently cleaved by $\beta$-secretase BACE1 and $\gamma$-secretase leading to toxic $\mathrm{A} \beta$-production of different peptide lengths (reviewed in $\mathrm{Bu}, 2009$ ). Importantly, Y27632-mediated ROCK inhibition changes $\gamma$-secretase cleavage specificity thereby shifting toxic $A \beta_{1-42}$-peptide production to $A \beta_{1-38}$ (Zhou et al., 2003), which is less toxic and might even be protective in combination with $A \beta_{1-42}$ (Vandersteen et al., 2012). However, total A $\beta$-production was not changed. Interestingly, ROCK-isoforms differentially act on the amyloidogenic pathway: While ROCK1 knock-down enhances total $\mathrm{A} \beta$-production in vitro, ROCK2 inhibition by the isoform-specific compound SR3677 reduced total A $\beta$-levels in vitro and in vivo (Herskowitz et al., 2013). Mechanistically, the authors suggested a regulation of BACE1-activity by ROCK2-mediated phosphorylation in combination with ROCK2-mediated APP phosphorylation triggering its processing (Herskowitz et al., 2013). In summary, ROCK-inhibition demonstrated efficacy in an AD-rat model most probably by inhibiting neuroinflammation. However, ROCK2-specific inhibition might be even more promising specifically targeting amyloidogenic pathways. In this context, ROCK1-inibition even might have a detrimental effect by enhancing $A \beta$ levels. Thus, beneficial effects of global ROCK inhibition reducing neuroinflammation but enhancing $A \beta$ biosynthesis via ROCK1 have to be trade-off against a specific ROCK2-inhibition without targeting glial cells. 


\section{Huntington's Disease (HD)}

$\mathrm{HD}$ is a hereditary neurodegenerative disease caused by CAGrepeats within the huntingtin ( $\mathrm{Htt})$-gene (The Huntington's Disease Collaborative Research Group, 1993). Thereby, the number of CAG-repeats correlates with the clinical onset which typically manifests by involuntary movements, and increasing motor- and cognitive dysfunctions. Consistent with the clinical picture, neuronal subpopulations display a selective vulnerability in HD with atrophy and neuronal degeneration in basal ganglia and cortex (reviewed in Shoulson and Young, 2011). The exact mechanism of neurodegeneration still remains unknown. However, mutant huntingtin aggregates or oligomers within neurons might play a crucial role (Legleiter et al., 2010).

Interestingly, the ROCK-pathway is directly involved in mutant huntingtin degradation and aggregate formation: Y27632 enhanced Htt-degradation thereby reducing its aggregation in vitro (Bauer et al., 2009), possibly by proteasomeactivation and macroautophagy (Bauer and Nukina, 2009). Moreover, Htt aggregation is facilitated by ROCK-dependent phosphorylation of vimentin which finally leads to enhanced levels of free IP3R1. As IP3R1 facilitates mutant htt-aggregation, the authors proposed a model where ROCK-inhibition inhibits htt-aggregation via vimentin-IP3R1 axis (Bauer et al., 2011, 2012). Alternatively, a mechanism based on a direct molecular interaction of Htt was proposed: The ROCK-downstream target profilin-1, an actin binding protein and a major regulator of actin-dynamics, as well as the neuronal isoform profilin2a, directly interact with huntingtin (Goehler et al., 2004; Shao et al., 2008). This interaction prevented Htt-aggregation indicating a beneficial effect in HD. Importantly, ROCKmediated phosphorylation of profilin at Serine-137 inhibited its aggregation suppressing activity, suggesting beneficial effects of ROCK-inhibition in HD mediated by profilindephosphorylation (Shao et al., 2008). Consistently, Y27632 partially rescued photoreceptor neurons in a HD-Drosophila model (Pollitt et al., 2003). However, ambiguous results were obtained in R6/2 HD-model mice. Y27632 only mildly improved motor-performance, but had no effect on lifespan, cellular atrophy or aggregate formation (Li et al., 2009b). This lack of effects might be due to an insufficient dosing-regimen as there was no impact on profilin-phosphorylation ( $\mathrm{Li}$ et al., 2009b). Intravitreal Fasudil application in the same model reduced retinal phospho-profilin1 staining and partially rescued retinal dysfunction ( $\mathrm{Li}$ et al., 2013). Although retinal dysfunction might not be a feature of HD (Petrasch-Parwez et al., 2005), ROCK inhibition therefore proofed its beneficial potential on the functional level. However, mechanisms underlying those effects remain unclear as Htt-aggregation state, neuroinflammation, neuronal survival, or axonal regeneration have not been measured in a ROCK-inhibited HD-mouse model yet. Interestingly, neuronal survival and axonal regeneration in a primary HD in vitro-model could both be rescued by ROCK-inhibition (Deyts et al., 2009) suggesting the inclusion of such outcomes in future in vivo studies.

\section{Spinal Muscular Atrophy (SMA)}

SMA is a monogenic motoneuron-disease affecting lower motoneurons progressively leading to paralysis, muscle atrophy and -in severe cases- to death. SMA is caused by deletions or loss-of-function mutations of the survival of motoneuron 1 (Smn1)-gene (Lefebvre et al., 1995). Humans harbor a second gene, Smn2, coding for the same protein. However, only low amounts of functional full-length protein is expressed from Smn2 (Lorson et al., 1999). While complete SMN-loss is embryonically lethal, low SMN-levels lead to SMA and consistently, the number of Smn2-gene copies inversely correlates with disease severity (Taylor et al., 1998). The SMN-protein is ubiquitously expressed, and not surprisingly severe SMA with low SMN levels is considered not to be of pure motoneuron-pathology rather than being a multi-system disorder (Shababi et al., 2014). However, motoneurons are still preferentially affected, arguing in favor of focusing on pathomechanisms specific for these cell-types. The role of ROCK in SMA in different cellular contexts has been extensively studied and already excellently reviewed (Coque et al., 2014), so that we will address this only at a glance.

Importantly, ROCK-activity is enhanced in SMA-mouse model spinal cords and inhibition of ROCK by Y27632 or Fasudil significantly enhanced survival, rescued neuromuscular junction (NMJ) morphology, and muscle atrophy (Bowerman et al., 2010, 2012). Consistently, neuronal SMA-in vitro models displayed an hyper-active ROCK-signaling axis, indicating a neuron-intrinsic mechanism (Bowerman et al., 2007; Nolle et al., 2011; Hensel et al., 2014). Based on a direct interaction of the SMN-protein with the ROCK-downstream target profilin2a, the neuronal profilin-isoform, we proposed a molecular mechanism of neurotoxicity induced by SMN-depletion (Nolle et al., 2011): SMN-reduction leads to changed profilin2a-homeostasis and an enhanced profilin2a-ROCK binding. This finally results in a hyper-phosphorylation of profilin2a and a sequestration of ROCK from other downstream-targets such as cofilin or myosin light chain phosphatase (MLCP). Accordingly, those ROCK-targets were hypo-phosphorylated and actin-composition changed towards more stable, less dynamic, filamentous (F) actin (Nolle et al., 2011). This might have important implications for neuronal motility processes based on actomyosin contractility. Accordingly, neurite outgrowth defects were reported in SMA in vitro models (Van Bergeijk et al., 2006; Nolle et al., 2011). However, involvement of other actin-based motility processes such as axonal transport or synaptic vesicle cycling is possible. In this model, beneficial effects of ROCK-inhibition are mainly mediated by reduced phosphorylation of neuronal profilin, however, positive effects on gliosis which is a well-known feature of SMA (Simic, 2008) is likely possible.

Besides an up-regulated ROCK-pathway, also the ERKsignaling axis is hyper-activated in SMA model mice spinal cords (Branchu et al., 2013). In contrast to the classical view of ERK as an anti-apoptotic, neurotrophic pathway this over-activation acts detrimental on SMA-phenotype as ERK-inhibition by the small inhibitor Selumetinib enhanced survival of SMA-mice (Branchu et al., 2013). Therefore, the authors suggested an SMA-specific mechanism based on elevated SMN-expression. According to 
the in vivo situation, we could confirm ERK hyper-activation in motoneuron-like cells in vitro (Hensel et al., 2012, 2014). Moreover, ROCK and ERK are bi-directionally linked, as ROCK inhibits ERK and vice versa (Hensel et al., 2014). This crosstalk was observed in a timeframe of minutes making it unlikely that expressional changes represent the underlying mechanism. Interestingly, the crosstalk became uni-directionalized in SMAmodel cells, as ERK was not capable to down-regulate ROCK selectively in SMA-cells. In summary, this leads to a cohyperactivation of the ROCK- and ERK-signaling axes in SMAcells (Hensel et al., 2014). As both pathways are potential therapeutic targets, we suggested a ROCK, ERK co-inhibition using a combination of Fasudil and Selumetinib. As detrimental ERK-hyper-activation could potentially be enhanced by ROCKinhibition via ROCK-ERK crosstalk, we even expect synergistic effects of a combined treatment approach (Hensel et al., 2014).

Another crosstalk partner of ROCK is the phosphatase and tensin homologue deleted on chromosome ten (PTEN). PTEN mainly antagonizes the phosphatidylinositol 3 kinase (PI3K) pathway by hydrolyzing the second messenger phosphatidylinositol (3,4,5)-triphosphate (PIP3) thereby blocking the recruitment and activation of Akt (reviewed in Song et al., 2012; Hopkins and Parsons, 2014). It was shown that ROCK directly interacts and phosphorylates PTEN at several sites (Li et al., 2005; Hopkins et al., 2014). In non-neuronal cells, activated ROCK1 induced PTEN-activity resulting in an inactivation of Akt ( $\mathrm{Li}$ et al., 2014a). Interestingly, in vitro data revealed that ROCK2, the neuronal ROCK-isoform, more potently phosphorylates PTEN than ROCK1 (Song et al., 2009). Thus, neuron-intrinsic ROCK-Akt crosstalk might be mediated via PTEN and enhanced ROCK-activities in SMA might lead to reduced Akt-activation via PTEN. However, while reduced Akt-activation has been pre-symptomatically observed in SMA-mice spinal cords (Branchu et al., 2013), to our knowledge, PTEN activity has not been monitored in SMA models so far. Importantly, increasing evidence has occurred about the role of PTEN in SMA: Knock-down of PTEN in cultured motoneurons as well as in SMA mice increases activity of the Akt-pathway, decreases disease severity and moderately enhances survival of SMA mice (Ning et al., 2010; Little et al., 2015).

\section{Amyotrophic Lateral Sclerosis (ALS)}

ALS is a motoneuron disease not only affecting lower motoneurons in spinal cord but also leading to upper motoneuron and brainstem degeneration. Clinical symptoms include muscle weakness and paralysis leading to death 3-5 years after onset, which typically develops in advanced adulthood (reviewed in Chen et al., 2013b). About 90\% of cases are sporadic with an unknown etiology; however, several risk genes with different penetrance have already been identified (Chen et al., 2013b). Mutant Superoxide dismutase 1 (SOD1) was the first gene identified to be involved in familiar ALS (Rosen, 1993), and the high penetrance allele SOD1 G93A (Synofzik et al., 2010) has been employed to generate the most extensively used ALS-mouse model (Gurney, 1997).

Interestingly, SOD $1^{\mathrm{G} 93 \mathrm{~A}}$-mice exhibited an enhanced ROCKactivity, elevated PTEN-activity while the Akt-pathway was down-regulated. Moreover, ROCK-inhibition by Fasudiltreatment completely rescued PTEN/Akt activity (Takata et al., 2013). This was accompanied by delayed symptom onset, enhanced survival and a partial rescue of motoneuronnumbers within spinal cord of SOD $1^{\mathrm{G} 93 \mathrm{~A}}$-mice (Takata et al., 2013). Interestingly, Fasudil-application in another study not only enhanced survival but also motor functions in $\mathrm{SOD} 1^{\mathrm{G} 93 \mathrm{~A}}$-mice. Moreover, motoneuron degeneration and nerve fibers were partially rescued (Tonges et al., 2014a). While the numbers of astroglial cells was reduced, Fasudil-treatment further enhanced microglia cell numbers but shifted morphology to the beneficial M2-microglia mechanism (Tonges et al., 2014a), which is in accordance with in vitro data (Zhang et al., 2013). Thus, two different studies suggested different mechanisms underlying beneficial effects of ROCK-inhibition in ALS-mouse models: (i) Altering the microglia-subtype composition, or (ii) inducing antiapoptotic signaling via non-canonical ROCK-crosstalk. However, also neuron-intrinsic canonical ROCK-signaling, controlling axonal regeneration, is a candidate-mechanism. Involvement of ROCK-pathway and actin-dynamics in ALS-pathogenesis has recently been supported by newly identified familial ALS-genes: Mutations in the profilin1gene are associated with high risks to develop ALS (Wu et al., 2012). Among those, profilin $1^{\text {T109M }}$ represents a phospho-site mutation (Ingre et al., 2013) indicating an important role of profilin-phosphorylation However, it is unclear whether ROCK is an upstream-kinase for this site.

\section{Summary and Future Perspectives}

Taken together, ROCK-inhibition ameliorates clinically relevant outcomes in rodent models of several NDDs making it a promising future treatment-strategy. However, with regard to chronic diseases detrimental long-term effects have to be considered highlighting alternative approaches such as nonsystemic application routes as well as ROCK-isoform specific inhibition. With regard to the underlying mechanisms, neurons as well as neuroinflammatory cells such as microglia seem to be involved in mediating the beneficial effects of ROCK-inhibition. Genetic models with cell specific ROCK-depletion would greatly improve our understanding of the exact contribution by different cell-types. Moreover, little is known about the ROCKdependent activation-mechanisms of neuroinflammatory cells on the molecular level. While ROCK-crosstalk with neurotrophic pathways is a relevant mechanism in neurons, such mechanisms have not been demonstrated in microglia yet. At this point, it has to been considered that elucidiation and analyses of single pathways and crosstalking molecules may limit our view on regulatory mechanisms. Instead, future work has to determine important nodes and edges as basic components of a molecular network with ROCK at its center. For example, network analyses could reveal new feedback loops and other network motifs relevant to determine direct and indirect effects of inhibitory drugs. Such a network should include multi-dimensional data on expression as well as on activation states to truly understand the flow of information in physiological and diseased conditions. 


\section{Acknowledgments}

Work of the Claus lab for elucidation of ROCK-inhibition is supported by the German Muscular Foundation (Deutsche Muskelstiftung) and by the Deutsche Gesellschaft für

\section{References}

Barcia, C., Ros, C. M., Annese, V., Carrillo-De Sauvage, M. A., Ros-Bernal, F., Gomez, A., et al. (2012). ROCK/Cdc42-mediated microglial motility and gliapse formation lead to phagocytosis of degenerating dopaminergic neurons in vivo. Sci. Rep. 2, 809. doi: 10.1038/srep00809

Bauer, P. O., Hudec, R., Goswami, A., Kurosawa, M., Matsumoto, G., Mikoshiba, K., et al. (2012). ROCK-phosphorylated vimentin modifies mutant huntingtin aggregation via sequestration of IRBIT. Mol. Neurodegener. 7, 43. doi: 10.1186/1750-1326-7-43

Bauer, P. O., Hudec, R., Ozaki, S., Okuno, M., Ebisui, E., Mikoshiba, K., et al. (2011). Genetic ablation and chemical inhibition of IP3R1 reduce mutant huntingtin aggregation. Biochem. Biophys. Res. Commun. 416, 13-17. doi: 10.1016/j.bbrc.2011.10.096

Bauer, P. O., and Nukina, N. (2009). Enhanced degradation of mutant huntingtin by rho kinase inhibition is mediated through activation of proteasome and macroautophagy. Autophagy 5, 747-748. doi: 10.4161/auto.5.5.8704

Bauer, P. O., Wong, H. K., Oyama, F., Goswami, A., Okuno, M., Kino, Y., et al. (2009). Inhibition of Rho kinases enhances the degradation of mutant huntingtin. J. Biol. Chem. 284, 13153-13164. doi: 10.1074/jbc.M809229200

Baxter, A. G. (2007). The origin and application of experimental autoimmune encephalomyelitis. Nat. Rev. Immunol. 7, 904-912. doi: 10.1038/nri2190

Bernhart, E., Kollroser, M., Rechberger, G., Reicher, H., Heinemann, A., Schratl, P., et al. (2010). Lysophosphatidic acid receptor activation affects the C13NJ microglia cell line proteome leading to alterations in glycolysis, motility, and cytoskeletal architecture. Proteomics 10, 141-158. doi: 10.1002/pmic.200900195

Bloom, G. S. (2014). Amyloid-beta and tau: the trigger and bullet in Alzheimer disease pathogenesis. JAMA Neurol. 71, 505-508. doi: 10.1001/jamaneurol.2013.5847

Borrajo, A., Rodriguez-Perez, A. I., Villar-Cheda, B., Guerra, M. J., and Labandeira-Garcia, J. L. (2014). Inhibition of the microglial response is essential for the neuroprotective effects of Rho-kinase inhibitors on MPTP-induced dopaminergic cell death. Neuropharmacology 85, 1-8. doi: 10.1016/j.neuropharm.2014.05.021

Bowerman, M., Beauvais, A., Anderson, C. L., and Kothary, R. (2010). Rho-kinase inactivation prolongs survival of an intermediate SMA mouse model. Hum. Mol. Genet. 19, 1468-1478. doi: 10.1093/hmg/ddq021

Bowerman, M., Murray, L. M., Boyer, J. G., Anderson, C. L., and Kothary, R. (2012). Fasudil improves survival and promotes skeletal muscle development in a mouse model of spinal muscular atrophy. BMC Med. 10:24. doi: 10.1186/1741-7015-10-24

Bowerman, M., Shafey, D., and Kothary, R. (2007). Smn depletion alters profilin II expression and leads to upregulation of the RhoA/ROCK pathway and defects in neuronal integrity. J. Mol. Neurosci. 32, 120-131. doi: 10.1007/s12031-0070024-5

Branchu, J., Biondi, O., Chali, F., Collin, T., Leroy, F., Mamchaoui, K., et al. (2013). Shift from extracellular signal-regulated kinase to AKT/cAMP response element-binding protein pathway increases survival-motor-neuron expression in spinal-muscular-atrophy-like mice and patient cells. J. Neurosci. 33, 4280-4294. doi: 10.1523/JNEUROSCI.2728-12.2013

$\mathrm{Bu}, \mathrm{G}$. (2009). Apolipoprotein $\mathrm{E}$ and its receptors in Alzheimer's disease: pathways, pathogenesis and therapy. Nat. Rev. Neurosci. 10, 333-344. doi: 10.1038/nrn2620

Chen, M., Liu, A., Ouyang, Y., Huang, Y., Chao, X., and Pi, R. (2013a). Fasudil and its analogs: a new powerful weapon in the long war against central nervous system disorders? Expert Opin. Investig. Drugs 22, 537-550. doi: 10.1517/13543784.2013.778242

Chen, S., Sayana, P., Zhang, X., and Le, W. (2013b). Genetics of amyotrophic lateral sclerosis: an update. Mol. Neurodegener. 8, 28. doi: 10.1186/1750-1326-8-28
Muskelkranke (DGM). NH has been supported by the Initiative SMA of the Gesellschaft für Muskelkranke (DGM). This review has been supported by the Niedersachsen-Research Network on Neuroinfectiology (N-RENNT) of the Ministry of Science and Culture of Lower Saxony.

Coque, E., Raoul, C., and Bowerman, M. (2014). ROCK inhibition as a therapy for spinal muscular atrophy: understanding the repercussions on multiple cellular targets. Front. Neurosci. 8:271. doi: 10.3389/fnins.2014.00271

Dauer, W., Kholodilov, N., Vila, M., Trillat, A. C., Goodchild, R., Larsen, K. E., et al. (2002). Resistance of alpha -synuclein null mice to the parkinsonian neurotoxin MPTP. Proc. Natl. Acad. Sci. U.S.A. 99, 14524-14529. doi: $10.1073 /$ pnas. 172514599

Deyts, C., Galan-Rodriguez, B., Martin, E., Bouveyron, N., Roze, E., Charvin, D., et al. (2009). Dopamine D2 receptor stimulation potentiates PolyQHuntingtin-induced mouse striatal neuron dysfunctions via Rho/ROCK-II activation. PLoS ONE 4:e8287. doi: 10.1371/journal.pone.0008287

Duffy, S. S., Lees, J. G., and Moalem-Taylor, G. (2014). The contribution of immune and glial cell types in experimental autoimmune encephalomyelitis and multiple sclerosis. Mult. Scler. Int. 2014, 285245. doi: 10.1155/2014/285245

Gibb, W. R., and Lees, A. J. (1989). The significance of the Lewy body in the diagnosis of idiopathic Parkinson's disease. Neuropathol. Appl. Neurobiol. 15, 27-44. doi: 10.1111/j.1365-2990.1989.tb01147.x

Goehler, H., Lalowski, M., Stelzl, U., Waelter, S., Stroedicke, M., Worm, U., et al. (2004). A protein interaction network links GIT1, an enhancer of huntingtin aggregation, to Huntington's disease. Mol. Cell 15, 853-865. doi: 10.1016/j.molcel.2004.09.016

Goldenberg, M. M. (2012). Multiple sclerosis review. P T 37, 175-184.

Gomez, T. M., and Letourneau, P. C. (2014). Actin dynamics in growth cone motility and navigation. J. Neurochem. 129, 221-234. doi: 10.1111/jnc.12506

Gurney, M. E. (1997). The use of transgenic mouse models of amyotrophic lateral sclerosis in preclinical drug studies. J. Neurol. Sci. 152(Suppl. 1), S67-S73. doi: 10.1016/s0022-510x(97)00247-5

Haass, C., Kaether, C., Thinakaran, G., and Sisodia, S. (2012). Trafficking and proteolytic processing of APP. Cold Spring Harb. Perspect. Med. 2:a006270. doi: 10.1101/cshperspect.a006270

Hensel, N., Ratzka, A., Brinkmann, H., Klimaschewski, L., Grothe, C., and Claus, P. (2012). Analysis of the fibroblast growth factor system reveals alterations in a mouse model of spinal muscular atrophy. PLoS ONE 7:e31202. doi: 10.1371/journal.pone.0031202

Hensel, N., Stockbrugger, I., Rademacher, S., Broughton, N., Brinkmann, H., Grothe, C., et al. (2014). Bilateral crosstalk of rho- and extracellularsignal-regulated-kinase (ERK) pathways is confined to an unidirectional mode in spinal muscular atrophy (SMA). Cell. Signal. 26, 540-548. doi: 10.1016/j.cellsig.2013.11.027

Herskowitz, J. H., Feng, Y., Mattheyses, A. L., Hales, C. M., Higginbotham, L. A., Duong, D. M., et al. (2013). Pharmacologic inhibition of ROCK2 suppresses amyloid-beta production in an Alzheimer's disease mouse model. J. Neurosci. 33, 19086-19098. doi: 10.1523/JNEUROSCI.2508-13.2013

Hopkins, B. D., Hodakoski, C., Barrows, D., Mense, S. M., and Parsons, R. E. (2014). PTEN function: the long and the short of it. Trends Biochem. Sci. 39, 183-190. doi: 10.1016/j.tibs.2014.02.006

Hopkins, B. D., and Parsons, R. E. (2014). Molecular pathways: intercellular PTEN and the potential of PTEN restoration therapy. Clin. Cancer Res. 20, 5379-5383. doi: 10.1158/1078-0432.CCR-13-2661

Hou, S. W., Liu, C. Y., Li, Y. H., Yu, J. Z., Feng, L., Liu, Y. T., et al. (2012). Fasudil ameliorates disease progression in experimental autoimmune encephalomyelitis, acting possibly through antiinflammatory effect. CNS Neurosci. Ther. 18, 909-917. doi: 10.1111/cns.12002

Huesa, G., Baltrons, M. A., Gomez-Ramos, P., Moran, A., Garcia, A., Hidalgo, J. et al. (2010). Altered distribution of RhoA in Alzheimer's disease and AbetaPP overexpressing mice. J. Alzheimers. Dis. 19, 37-56. doi: 10.3233/JAD-2010-1203

Ingre, C., Landers, J. E., Rizik, N., Volk, A. E., Akimoto, C., Birve, A., et al. (2013). A novel phosphorylation site mutation in profilin 1 revealed in a large screen of US, Nordic, and German amyotrophic lateral 
sclerosis/frontotemporal dementia cohorts. Neurobiol. Aging 34, 1708 e1701e1706. doi: 10.1016/j.neurobiolaging.2012.10.009

International Multiple Sclerosis Genetics et al., C., Wellcome Trust Case Control, C., Sawcer, S., Hellenthal, G., Pirinen, M., Spencer, C. C., et al. (2011). Genetic risk and a primary role for cell-mediated immune mechanisms in multiple sclerosis. Nature 476, 214-219. doi: 10.1038/nature10251

Koch, J. C., Tonges, L., Michel, U., Bahr, M., and Lingor, P. (2014). Viral vector-mediated downregulation of RhoA increases survival and axonal regeneration of retinal ganglion cells. Front. Cell. Neurosci. 8:273. doi: $10.3389 /$ fncel.2014.00273

Lefebvre, S., Burglen, L., Reboullet, S., Clermont, O., Burlet, P., Viollet, L., et al. (1995). Identification and characterization of a spinal muscular atrophydetermining gene. Cell 80, 155-165. doi: 10.1016/0092-8674(95)90460-3

Legleiter, J., Mitchell, E., Lotz, G. P., Sapp, E., Ng, C., Difiglia, M., et al. (2010). Mutant huntingtin fragments form oligomers in a polyglutamine lengthdependent manner in vitro and in vivo. J. Biol. Chem. 285, 14777-14790. doi: 10.1074/jbc.M109.093708

Li, G., Liu, L., Shan, C., Cheng, Q., Budhraja, A., Zhou, T., et al. (2014a). RhoA/ROCK/PTEN signaling is involved in AT-101-mediated apoptosis in human leukemia cells in vitro and in vivo. Cell Death Dis. 5, e998. doi: 10.1038/cddis.2013.519

Li, L. H., Qin, H. Z., Wang, J. L., Wang, J., Wang, X. L., and Gao, G. D. (2009a). Axonal degeneration of nigra-striatum dopaminergic neurons induced by 1-methyl-4-phenyl-1,2,3,6-tetrahydropyridine in mice. J. Int. Med. Res. 37, 455-463. doi: 10.1177/147323000903700221

Li, M., Huang, Y., Ma, A. A., Lin, E., and Diamond, M. I. (2009b). Y-27632 improves rotarod performance and reduces huntingtin levels in R6/2 mice. Neurobiol. Dis. 36, 413-420. doi: 10.1016/j.nbd.2009.06.011

Li, M., Yasumura, D., Ma, A. A., Matthes, M. T., Yang, H., Nielson, G., et al. (2013). Intravitreal administration of HA-1077, a ROCK inhibitor, improves retinal function in a mouse model of huntington disease. PLOS ONE 8:e56026. doi: 10.1371/journal.pone.0056026

Li, Y. H., Yu, J. Z., Liu, C. Y., Zhang, H., Zhang, H. F., Yang, W. F., et al. (2014b). Intranasal delivery of FSD-C10, a novel Rho kinase inhibitor, exhibits therapeutic potential in experimental autoimmune encephalomyelitis. Immunology 143, 219-229. doi: 10.1111/imm.12303

Li, Z., Dong, X., Wang, Z., Liu, W., Deng, N., Ding, Y., et al. (2005). Regulation of PTEN by Rho small GTPases. Nat. Cell Biol. 7, 399-404. doi: 10.1038/ncb1236

Lin, W. F., Chen, C. J., Chang, Y. J., Chen, S. L., Chiu, I. M., and Chen, L. (2009). SH2B1beta enhances fibroblast growth factor 1 (FGF1)-induced neurite outgrowth through MEK-ERK1/2-STAT3-Egr1 pathway. Cell. Signal. 21, 1060-1072. doi: 10.1016/j.cellsig.2009.02.009

Lingor, P., Tonges, L., Pieper, N., Bermel, C., Barski, E., Planchamp, V., et al. (2008). ROCK inhibition and CNTF interact on intrinsic signalling pathways and differentially regulate survival and regeneration in retinal ganglion cells. Brain 131, 250-263. doi: 10.1093/brain/awm284

Little, D., Valori, C. F., Mutsaers, C. A., Bennett, E. J., Wyles, M., Sharrack, B., et al. (2015). PTEN depletion decreases disease severity and modestly prolongs survival in a mouse model of spinal muscular atrophy. Mol. Ther. 23, 270-277. doi: $10.1038 / \mathrm{mt} .2014 .209$

Lorson, C. L., Hahnen, E., Androphy, E. J., and Wirth, B. (1999). A single nucleotide in the SMN gene regulates splicing and is responsible for spinal muscular atrophy. Proc. Natl. Acad. Sci. U.S.A. 96, 6307-6311. doi: 10.1073/pnas.96.11.6307

Lowery, L. A., and Van Vactor, D. (2009). The trip of the tip: understanding the growth cone machinery. Nat. Rev. Mol. Cell Biol. 10, 332-343. doi: $10.1038 /$ nrm 2679

Monnier, P. P., Sierra, A., Schwab, J. M., Henke-Fahle, S., and Mueller, B. K. (2003). The Rho/ROCK pathway mediates neurite growth-inhibitory activity associated with the chondroitin sulfate proteoglycans of the CNS glial scar. Mol. Cell. Neurosci. 22, 319-330. doi: 10.1016/\$1044-7431(02)00035-0

Moon, M. Y., Kim, H. J., Li, Y., Kim, J. G., Jeon, Y. J., Won, H. Y., et al. (2013). Involvement of small GTPase RhoA in the regulation of superoxide production in BV2 cells in response to fibrillar Abeta peptides. Cell. Signal. 25, 1861-1869. doi: 10.1016/j.cellsig.2013.05.023

Moreno-Trevino, M. G., Castillo-Lopez, J., and Meester, I. (2015). Moving away from amyloid Beta to move on in Alzheimer research. Front. Aging Neurosci. 7:2. doi: 10.3389/fnagi.2015.00002
Ning, K., Drepper, C., Valori, C. F., Ahsan, M., Wyles, M., Higginbottom, A., et al. (2010). PTEN depletion rescues axonal growth defect and improves survival in SMN-deficient motor neurons. Hum. Mol. Genet. 19, 3159-3168. doi: $10.1093 / \mathrm{hmg} / \mathrm{ddq} 226$

Nishioka, K., Hayashi, S., Farrer, M. J., Singleton, A. B., Yoshino, H., Imai, H., et al. (2006). Clinical heterogeneity of alpha-synuclein gene duplication in Parkinson's disease. Ann. Neurol. 59, 298-309. doi: 10.1002/ana.20753

Nolle, A., Zeug, A., Van Bergeijk, J., Tonges, L., Gerhard, R., Brinkmann, H., et al. (2011). The spinal muscular atrophy disease protein SMN is linked to the Rho-kinase pathway via profilin. Hum. Mol. Genet. 20, 4865-4878. doi: $10.1093 / \mathrm{hmg} / \mathrm{ddr} 425$

Ortiz, G. G., Pacheco-Moises, F. P., Macias-Islas, M. A., Flores-Alvarado, L. J., Mireles-Ramirez, M. A., Gonzalez-Renovato, E. D., et al. (2014). Role of the blood-brain barrier in multiple sclerosis. Arch. Med. Res. 45, 687-697. doi: 10.1016/j.arcmed.2014.11.013

Perl, D. P. (2010). Neuropathology of Alzheimer's disease. Mt. Sinai J. Med. 77, 32-42. doi: $10.1002 / \mathrm{msj} .20157$

Petrasch-Parwez, E., Saft, C., Schlichting, A., Andrich, J., Napirei, M., Arning, L., et al. (2005). Is the retina affected in Huntington disease? Acta Neuropathol. 110, 523-525. doi: 10.1007/s00401-005-1092-7

Pollitt, S. K., Pallos, J., Shao, J., Desai, U. A., Ma, A. A., Thompson, L. M., et al. (2003). A rapid cellular FRET assay of polyglutamine aggregation identifies a novel inhibitor. Neuron 40, 685-694. doi: 10.1016/S0896-6273(03)00697-4

Polymeropoulos, M. H., Lavedan, C., Leroy, E., Ide, S. E., Dehejia, A., Dutra, A., et al. (1997). Mutation in the alpha-synuclein gene identified in families with Parkinson's disease. Science 276, 2045-2047. doi: $10.1126 /$ science. 276.5321 .2045

Ponomarev, E. D., Shriver, L. P., Maresz, K., and Dittel, B. N. (2005). Microglial cell activation and proliferation precedes the onset of CNS autoimmunity. J. Neurosci. Res. 81, 374-389. doi: 10.1002/jnr.20488

Rosen, D. R., Siddique, T., Patterson, D., Figlewicz, D. A., Sapp, P., Hentati, A., et al. (1993). Mutations in $\mathrm{Cu} / \mathrm{Zn}$ superoxide dismutase gene are associated with familial amyotrophic lateral sclerosis. Nature 362, 59-62. doi: $10.1038 / 362059 \mathrm{a} 0$

Saal, K. A., Koch, J. C., Tatenhorst, L., Szego, E. M., Ribas, V. T., Michel, U., et al. (2015). AAV.shRNA-mediated downregulation of ROCK2 attenuates degeneration of dopaminergic neurons in toxin-induced models of Parkinson's disease in vitro and in vivo. Neurobiol. Dis. 73, 150-162. doi: 10.1016/j.nbd.2014.09.013

Schiesling, C., Kieper, N., Seidel, K., and Kruger, R. (2008). Review: familial Parkinson's disease-genetics, clinical phenotype and neuropathology in relation to the common sporadic form of the disease. Neuropathol. Appl. Neurobiol. 34, 255-271. doi: 10.1111/j.1365-2990.2008.00952.x

Selkoe, D. J. (2001). Alzheimer's disease results from the cerebral accumulation and cytotoxicity of amyloid beta-protein. J. Alzheimers. Dis. 3, 75-80.

Shababi, M., Lorson, C. L., and Rudnik-Schoneborn, S. S. (2014). Spinal muscular atrophy: a motor neuron disorder or a multi-organ disease? J. Anat. 224, 15-28. doi: $10.1111 /$ joa. 12083

Shao, J., Welch, W. J., Diprospero, N. A., and Diamond, M. I. (2008). Phosphorylation of profilin by ROCK1 regulates polyglutamine aggregation. Mol. Cell. Biol. 28, 5196-5208. doi: 10.1128/MCB.00079-08

Shoulson, I., and Young, A. B. (2011). Milestones in huntington disease. Mov. Disord. 26, 1127-1133. doi: 10.1002/mds.23685

Simic, G. (2008). Pathogenesis of proximal autosomal recessive spinal muscular atrophy. Acta Neuropathol. 116, 223-234. doi: 10.1007/s00401-0080411-1

Singleton, A. B., Farrer, M., Johnson, J., Singleton, A., Hague, S., Kachergus, J., et al. (2003). alpha-Synuclein locus triplication causes Parkinson's disease. Science 302, 841. doi: $10.1126 /$ science. 1090278

Song, M. S., Salmena, L., and Pandolfi, P. P. (2012). The functions and regulation of the PTEN tumour suppressor. Nat. Rev. Mol. Cell Biol. 13, 283-296. doi: $10.1038 / \mathrm{nrm} 3330$

Song, P., Zhang, M., Wang, S., Xu, J., Choi, H. C., and Zou, M. H. (2009). Thromboxane A2 receptor activates a Rho-associated kinase/LKB1/PTEN pathway to attenuate endothelium insulin signaling. J. Biol. Chem. 284, 17120-17128. doi: 10.1074/jbc.M109.012583

Song, Y., Chen, X., Wang, L. Y., Gao, W., and Zhu, M. J. (2013). Rho kinase inhibitor fasudil protects against beta-amyloid-induced hippocampal 
neurodegeneration in rats. CNS Neurosci. Ther. 19, 603-610. doi: $10.1111 / \mathrm{cns} .12116$

Spillantini, M. G., Schmidt, M. L., Lee, V. M., Trojanowski, J. Q., Jakes, R., and Goedert, M. (1997). Alpha-synuclein in Lewy bodies. Nature 388, 839-840. doi: $10.1038 / 42166$

Stys, P. K., Zamponi, G. W., Van Minnen, J., and Geurts, J. J. (2012). Will the real multiple sclerosis please stand up? Nat. Rev. Neurosci. 13, 507-514. doi: $10.1038 / \mathrm{nrn} 3275$

Sun, X., Minohara, M., Kikuchi, H., Ishizu, T., Tanaka, M., Piao, H., et al. (2006). The selective Rho-kinase inhibitor Fasudil is protective and therapeutic in experimental autoimmune encephalomyelitis. J. Neuroimmunol. 180, 126-134. doi: 10.1016/j.jneuroim.2006.06.027

Synofzik, M., Fernandez-Santiago, R., Maetzler, W., Schols, L., and Andersen, P. M. (2010). The human G93A SOD1 phenotype closely resembles sporadic amyotrophic lateral sclerosis. J. Neurol. Neurosurg. Psychiatry 81, 764-767. doi: 10.1136/jnnp.2009.181719

Tajouri, L., Mellick, A. S., Tourtellotte, A., Nagra, R. M., and Griffiths, L. R. (2005). An examination of MS candidate genes identified as differentially regulated in multiple sclerosis plaque tissue, using absolute and comparative real-time Q-PCR analysis. Brain Res. Brain Res. Protoc. 15, 79-91. doi: 10.1016/j.brainresprot.2005.04.003

Takata, M., Tanaka, H., Kimura, M., Nagahara, Y., Tanaka, K., Kawasaki, K., et al. (2013). Fasudil, a rho kinase inhibitor, limits motor neuron loss in experimental models of amyotrophic lateral sclerosis. Br. J. Pharmacol. 170, 341-351. doi: 10.1111/bph. 12277

Tang, Y., and Le, W. (2015). Differential Roles of M1 and M2 Microglia in Neurodegenerative Diseases. Mol. Neurobiol. doi: 10.1007/s12035-014-9070-5. [Epub ahead of print].

Tatenhorst, L., Tonges, L., Saal, K. A., Koch, J. C., Szego, E. M., Bahr, M., et al. (2014). Rho kinase inhibition by fasudil in the striatal 6-hydroxydopamine lesion mouse model of Parkinson disease. J. Neuropathol. Exp. Neurol. 73, 770-779. doi: 10.1097/NEN.0000000000000095

Taylor, J. E., Thomas, N. H., Lewis, C. M., Abbs, S. J., Rodrigues, N. R., Davies, K. E., et al. (1998). Correlation of SMNt and SMNc gene copy number with age of onset and survival in spinal muscular atrophy. Eur. J. Hum. Genet. 6, 467-474. doi: 10.1038/sj.ejhg. 5200210

The Huntington's Disease Collaborative Research Group (1993). A novel gene containing a trinucleotide repeat that is expanded and unstable on Huntington's disease chromosomes. Cell 72, 971-983.

Tonges, L., Frank, T., Tatenhorst, L., Saal, K. A., Koch, J. C., Szego, E. M., et al. (2012). Inhibition of rho kinase enhances survival of dopaminergic neurons and attenuates axonal loss in a mouse model of Parkinson's disease. Brain 135, 3355-3370. doi: 10.1093/brain/aws254

Tonges, L., Gunther, R., Suhr, M., Jansen, J., Balck, A., Saal, K. A., et al. (2014a). Rho kinase inhibition modulates microglia activation and improves survival in a model of amyotrophic lateral sclerosis. Glia 62, 217-232. doi: 10.1002/glia.22601

Tonges, L., Koch, J. C., Bahr, M., and Lingor, P. (2011). ROCKing Regeneration: Rho Kinase Inhibition as Molecular Target for Neurorestoration. Front. Mol. Neurosci. 4:39. doi: 10.3389/fnmol.2011.00039

Tonges, L., Szego, E. M., Hause, P., Saal, K. A., Tatenhorst, L., Koch, J. C., et al. (2014b). Alpha-synuclein mutations impair axonal regeneration in models of Parkinson's disease. Front. Aging Neurosci. 6:239. doi: 10.3389/fnagi.2014. 00239

Trapp, B. D., Peterson, J., Ransohoff, R. M., Rudick, R., Mork, S., and Bo, L. (1998). Axonal transection in the lesions of multiple sclerosis. N. Engl. J. Med. 338, 278-285. doi: 10.1056/NEJM199801293380502

Van Bergeijk, J., Haastert, K., Grothe, C., and Claus, P. (2006). Valproic acid promotes neurite outgrowth in PC12 cells independent from regulation of the survival of motoneuron protein. Chem. Biol. Drug Des. 67, 244-247. doi: 10.1111/j.1747-0285.2006.00369.x

Vandersteen, A., Masman, M. F., De Baets, G., Jonckheere, W., Van Der Werf, K., Marrink, S. J., et al. (2012). Molecular plasticity regulates oligomerization and cytotoxicity of the multipeptide-length amyloid-beta peptide pool. J. Biol. Chem. 287, 36732-36743. doi: 10.1074/jbc.M112.394635

Villar-Cheda, B., Dominguez-Meijide, A., Joglar, B., Rodriguez-Perez, A. I., Guerra, M. J., and Labandeira-Garcia, J. L. (2012). Involvement of microglial RhoA/Rho-kinase pathway activation in the dopaminergic neuron death. Role of angiotensin via angiotensin type 1 receptors. Neurobiol. Dis. 47, 268-279. doi: 10.1016/j.nbd.2012.04.010

Wu, C. H., Fallini, C., Ticozzi, N., Keagle, P. J., Sapp, P. C., Piotrowska, K., et al. (2012). Mutations in the profilin 1 gene cause familial amyotrophic lateral sclerosis. Nature 488, 499-503. doi: 10.1038/nature11280

Zhang, H., Li, Y., Yu, J., Guo, M., Meng, J., Liu, C., et al. (2013). Rho kinase inhibitor fasudil regulates microglia polarization and function. Neuroimmunomodulation 20, 313-322. doi: 10.1159/000351221

Zhou, Y., Su, Y., Li, B., Liu, F., Ryder, J. W., Wu, X., et al. (2003). Nonsteroidal anti-inflammatory drugs can lower amyloidogenic Abeta42 by inhibiting Rho. Science 302, 1215-1217. doi: 10.1126/science.1090154

Conflict of Interest Statement: The authors declare that the research was conducted in the absence of any commercial or financial relationships that could be construed as a potential conflict of interest.

Copyright (c) 2015 Hensel, Rademacher and Claus. This is an open-access article distributed under the terms of the Creative Commons Attribution License (CC BY). The use, distribution or reproduction in other forums is permitted, provided the original author(s) or licensor are credited and that the original publication in this journal is cited, in accordance with accepted academic practice. No use, distribution or reproduction is permitted which does not comply with these terms. 